\title{
Antibiotic resistance pattern of Staphylococcus aureus with reference to MRSA isolates from pediatric patients
}

\author{
Raja Ram Gurung ( $\sim$ nepaliraza07@gmail.com ) \\ Nepal Journal of Biotechnology https://orcid.org/0000-0002-4515-5853 \\ Prashanna Maharjan \\ Tribhuvan University- Amrit Science College
}

Ganga GC

Tribhuvan University - Trichandra Multiple Campus

\section{Research article}

Keywords: Methicillin-Resistant Staphylococcus aureus (MRSA), Macrolide-Lincosamide-Streptogramin B (MLSB) phenotype, Inducible Clindamycin Resistant (ICR) test or D-zone test, Antibiotic resistance, Nepal

Posted Date: July 1st, 2019

DOI: https://doi.org/10.21203/rs.2.10785/v1

License: (c) (i) This work is licensed under a Creative Commons Attribution 4.0 International License. Read Full License

Version of Record: A version of this preprint was published at Future Science OA on February 24th, 2020. See the published version at https://doi.org/10.2144/fsoa-2019-0122. 


\section{Abstract}

Background: Staphylococcus aureus is one of the important superbugs distributed throughout the world. It causes minor skin infections to severe complications including nosocomial infections in both hospitals and community settings. These strains have multi-drug resistant property. Hence, they are difficult to manage which increase health-related costs and simultaneously intensifying the need for new antibiotics. The extent of Methicillin-Resistant Staphylococcus aureus (MRSA) in children is largely unknown. The study determines the current status of $\mathrm{S}$. aureus and MRSA causing various infections in pediatric patients visiting International Friendship Children's Hospital (IFCH). Methods: A cross-sectional study was conducted among patients visiting a hospital. Various clinical specimens were aseptically collected and processed according to standard microbiological procedures. Isolation and identification of S. aureus were done by microscopy, mannitol fermentation, and coagulase positivity. All identified S. aureus isolates subjected to in-vitro antibiogram by Kirby-Bauer disc diffusion technique adopting Clinical and Laboratory Standards Institute (CLSI) guideline. Isolates resistant to cefoxitin were considered to be MRSA. Whereas, isolates produced D-shaped inhibition zone around clindamycin when kept near erythromycin were considered to be Inducible Clindamycin Resistant (ICR). Results: 672 various types of clinical samples were processed from the microbiology laboratory from June and November 2015. Out of 300 culture positive samples, $52(17.3 \%)$ were S. aureus isolates, among them $39(75.0 \%)$ were found to be MRSA. The D-test showed that Macrolide-Lincosamide-Streptogramin-B (MLSB) phenotype was $15.4 \%$. Conclusion: The study shows the MRSA occurrence is prevalent in pediatric patients and newer classes' drugs are found more effective than $\beta$-lactam drugs to treat $S$. aureus infection. However, restriction on the indiscriminate use of such drugs may be an effective strategy to control the drug resistance. Keywords: Methicillin-Resistant Staphylococcus aureus (MRSA), Macrolide-LincosamideStreptogramin B (MLSB) phenotype, Inducible Clindamycin Resistant (ICR) test or D-zone test, Antibiotic resistance, Nepal

\section{Background}

S. aureus remains ubiquitous in the environment and normal flora of animals. Theyare commensals as well as pathogenic bacteria [1]. It is known to occur as normal flora of the skin and is estimated that about $20 \%$ of the world population are persistent carriers. Moreover $60 \%$ population carries it occasionally during their life-time [2,3]. However, they are considered to be an opportunistic pathogen for human and animal if they get an opportunity to enter the bloodstream and tissue [1,2]. Many of them become infectious only when they get a chance to enter into the skin or mucous membrane punctured by any penetrating objects [4]. It is found to cause a range of illness from minor skin infections, such as pimples, impetigo, boils, cellulitis, scalded skin syndrome, folliculitis, furuncles, carbuncles, and abscesses to life-threatening diseases such as pneumonia, osteomyelitis, meningitis, Toxic Shock Syndrome (TSS), endocarditis and septicemia [5].

Methicillin-resistant Staphylococcus aureus (MRSA) is a strain of S. aureus that has acquired resistance to $\beta$-lactam antibiotics, which include penicillin and cephalosporin. MRSA strains are a versatile and most 
significant nosocomial pathogen, often causing postsurgical wound infections almost exclusively of hospital origin described in 1961 [6]. MRSA infections account for $20-80 \%$ of all nosocomial S. aureus infectionsin many centers across the world $[7,8]$ that lead to increased mortality, morbidity hospital stay, and costs $[9,10]$. WHO $(2018)$ have reported that $64 \%$ of MRSA infected patients are more likely to die than non MRSA infected patients [11]. MRSA may transmitfrom person to person by physical contact and rarely by air. The nasopharynx is the main ecological niche of the $S$. aureus [12], although, it is found in almost all body parts. With little change in overall mortality, the frequency of Community Acquired MRSA (CAMRSA) and Hospital Acquired MRSA (HAMRSA) infections have increased steadily. They contribute to the failure of empirical therapy. Treatment of these infections has become more difficult because of the emergence of multidrug-resistance (MDR) strains too [13]. MDR is defined as resistance to three or more antimicrobial classes. Multidrug-resistant $S$. aureus raises greater concern because of its high virulence capacity [14], high ability to cause a diverse array of life-threatening infections, and its capacity to adapt to different environmental conditions $[13,15]$. The emergence of resistant strains is contributing to nosocomial infections [16].

The emergence and the spread of the Multi Drug Resistant (MDR) Staphylococcus aureus bacteria is a global threat for the therapeutic management of staphylococcal infections. Studies have shown that asymptomatic carrier children are a potential vector for the dissemination of MRSA in the community. [17, 18]. However, to our knowledge, the exact extent of MRSA in children of Nepal has not yet been well assessed. Therefore, this study is an initiative to explore the distribution of MRSA in pediatric patients visiting IFCH. The presence of MRSA among a population of children were evaluated and assessed the antimicrobial susceptibility profile. The infection among inpatients with those of the outpatients was compared. The association between the causative organism with different variables such as age, gender, and type of samples were assessed. These are important factors in S. aureus related infections. We believe that this kind of study will be helping the physicians to administer the first line treatment by selecting appropriate therapeutic drug against $S$. aureus.

\section{Methods}

\section{Materials:}

All the chemical, kits, antibiotic discs and microbiological media were purchased from Himedia Pvt. Ltd. Co., Mumbai, India.

Sample collection: The study was conducted in the microbiology laboratory of IFCH, Kathmandu during the period of Jun 2016 to Nov 2016. A total of 672 various clinical specimens viz. blood, urine, stool, pus swab from an ear, eye, throat, vaginal, and body parts where burn or wound occurred were collected from both inpatients and outpatients. Only those patients who were not undertaking any medication and referred by a clinician were included in the study. Samples were stored in a sterile, leak-proof, screwcapped container with a proper label (Lab Id no., age, gender, date, time).

\section{Laboratory assessment}




\section{Isolation and Identification:}

The specimens were processed in the microbiology laboratory within two hrs. of the collection. A sample of blood was collected in the Brain Heart Infusion (BHI) broth in 1:10 v/v ratio immediately, mixed well and then screw tightened the $\mathrm{BHI}$ bottle. The $\mathrm{BHI}$ bottles were then incubated at $37^{\circ} \mathrm{C}$ for up to 72 hours followed by subculture on Blood Agar (BA) and MacConkey Agar (MA) plates. Samples such as pus, urine, stool etc. were directly inoculated into BA and MA, whereas, CSF samples were inoculated into MA and Chocolate (CA). Only the growth obtained in BA and CA plates were further cultured into Mannitol Salt Agar (MSA), an enrichment media for isolation of $S$. aureus. The BA and CA plates were incubated anaerobically $\left(5-10 \% \mathrm{CO}_{2}\right)$, while, MA and MSA plates were incubated aerobically at $37^{\circ} \mathrm{C}$ for 24 hours. Colonies formed in MSA were picked and processed for gram staining. Only cocci were processed for the identification of $S$. aureus. For conformational identification of $S$. aureuS; catalase test, coagulase (slide and tube) test were performed with the known positive and negative control strains (Fig. 1).

\section{Macroscopic examination:}

$\beta$-hemolytic activity was detected on BA medium while colony characteristics were examined on MSA and BA medium for the identification of all the isolates. (Fig. 2 and 3 ).

\section{Antibiotic susceptibility:}

All identified $S$. aureus isolates from various clinical specimens were subjected to in-vitro Antibiotic Susceptibility Test (AST) by Kirby-Bauer disc diffusion method following CLSI guidelines [19]. Following 13 antibiotics at the indicated concentrations were tested against all the strains: cefoxitin $(30 \mu)$, penicillin $(10 \mu)$, ofloxacin $(5 \mu)$, erythromycin $(15 \mu)$, amoxicillin/clavulanic acid $(15 \mu)$, ciprofloxacin $(5 \mu)$, cotrimoxazole $(25 \mu)$, ampicillin $(10 \mu)$, amoxicillin $(10 \mu)$, cloxacillin $(5 \mu)$, azithromycin $(15 \mu)$, tetracycline $(30 \mu)$, gentamicin $(10 \mu)$, and vancomycin $(30 \mu)$. For identification of MRSA, Cefoxitin disc $(30 \mu)$ was used. A zone of inhibition (ZOI) less than $22 \mathrm{~mm}$. or any discernible growth within ZOI by S. aureus against cefoxitin in MHA plate was indicative of methicillin resistance. S. aureus ATCC 25923 was used as a standard control strain. Methicillin resistance was tested for all the $S$. aureus isolates by agar screening method using Muller Hinton Agar [20] supplemented with $4 \% \mathrm{NaCl}$ against cefoxitin disc. Whereas for ICR identification, clindamycin and erythromycin drugs were kept apart 15-26 mm in MHA and D-shaped inhibition zone was observed around clindamycin (Fig. 4, 5 \& 6).

\section{Statistical analysis}

Microsoft Excel 2013 was used to record the laboratory data and statistical analysis software (IMB-SPSS V21.0) was used to calculate a $p$-value by using the Pearson Chi-Square test. $p>0.05$ was considered statistically significant at 95\% Confidence Interval (CI) (Table 2 \& 6).

\section{Results}




\section{Isolation of Staphylococcus aureus}

Out of 672 clinical samples processed, bacterial growth positive was detected in $300(44.64 \%)$ samples only. Among all growth positive samples, $S$. aureus isolates were positive in 52(17.3\%) samples, that constituted $29(55.7 \%)$ were from inpatient and 23(44.3\%) from outpatients (Table 1).

\section{Demography of $S$. aureus}

Among the 52 S. aureus positive samples, 35(67.3\%) and 17(32.7\%) were isolated from male and female gender respectively (Table 3 ). Distribution of $S$. aureus in the toddler age group is maximum i.e. 17(32.7\%) and least in adolescent 2(3.8\%) age group children in both genders (Table 1).

\section{Distribution of MRSA in outpatients and inpatients}

Addition to MRSA, response to cefoxitin was also stratified as Methicillin Intermediate S. aureus (MISA) and Methicillin Sensitive $S$. aureus (MSSA). Cefoxitin disc diffusion test showed that out of 52 S. aureus isolates, 39 (75.0\%), 7 (13.5\%) and $6(11.5 \%)$ were identified as MRSA, MISA, and MSSA respectively. Moreover, we found that among these $21(72.4 \%), 5(17.2 \%)$ and $3(10.3 \%)$ of MRSA, MISA, and MSSA respectively were in inpatients. The analysis further showed that the incidence of MRSA and MSSA isolations (78.3\%) and (13.0\%) respectively were slightly higher in outpatient samples than in the inpatient sample. However, the association between the MRSA occurrence and inpatients was statistically not significant $(p=0.637$ at $95 \% \mathrm{Cl})$. On the other hand, the incidence of MISA isolation was lower in inpatient samples 6 compare to outpatient samples $2(8.7 \%)$. This data clearly shows that the frequency of MRSA in inpatients is higher as compared to outpatient (Table 2).

\section{Distribution of $S$. aureus in the various infection group}

Of 35 samples from male patients, 23 (44.2\%) were from skin and soft tissue infection (SSTI) patients (skin swab), 7 (13.5\%) were from respiratory tract infection (RTI) patients (throat and nasal swabs), 5 $(9.6 \%)$ were blood samples, and none $(0 \%)$ were urine samples. Likewise, out of 17 samples from female patients, 11 (21.2\%) were from SSTI patients, 3 (5.77\%) were from RTI patients, 2 (3.9\%) were blood samples, and $1(1.9 \%)$ was urine sample. These results showed in both male and female pediatric patients, $S$. aureus strains were prevalent in SSTIs than compare to remaining other types of infections (Table 3).

\section{Antibiotic resistance pattern of Staphylococcus aureus}

The antimicrobial resistance pattern of MRSA and MSSA isolates against antimicrobial agents are summarized in (Table 4). More than 25\% of MRSA isolates were resistant to ampicillin, ciprofloxacin, cotrimoxazole, erythromycin, clindamycin, azithromycin and tetracycline. Only a few of the MRSA isolates were found to be resistant to vancomycin (0\%), cloxacillin (17.9\%), gentamycin $(20.68 \%$ ) amikacin (10.2\%) and chloramphenicol (23.1\%). $\beta$-lactam antibiotics like cefoxitin (100\%) and penicillin (100\%) resistance were found to be ineffective against MSSA too. While ampicillin (69.2\%), and ciprofloxacin 
(84.2\%) were shown resistance against MSSA isolates. Rest of the antibiotic showed less than $50 \%$ resistance towards the isolated MSSA.

\section{Susceptibility pattern of $S$. aureus towards $\mathrm{MLS}_{\mathrm{B}}$ antibiotics}

Among $52 \mathrm{~S}$. aureus isolates, inducible $\mathrm{MLS}_{\mathrm{B}}$ resistance, constitutive $\mathrm{MLS}_{\mathrm{B}}, \mathrm{MS}_{\mathrm{B}}$ and susceptibility was found in $8(15.4 \%), 7(13.5 \%), 17(32.7 \%)$ and $20(38.5 \%)$ respectively. Of the 39 MRSA isolates, $15.4 \%$ $(n=6 / 39)$ had inducible $M_{L} S_{B}$ resistance. Constitutive $M_{L} S_{B}$ was observed in $12.8 \%(n=5 / 39)$ of MRSA isolates. In $38.5 \%(n=15 / 39)$ of isolates, $M_{B}$ resistance was observed and in $33.3 \%(n=13 / 39)$ susceptibility to both erythromycin and clindamycin was observed. Out of 13 MSSA isolates, $38.5 \%$ $(n=5 / 13)$ were susceptible to both of erythromycin and clindamycin, 2(15.4\%) isolates each had inducible and constitutive $\mathrm{MLS}_{\mathrm{B}}$ resistance and 4 (30.8\%) isolates had $\mathrm{MS}_{\mathrm{B}}$ resistance. Most of the MSSA were sensitive to both erythromycin and clindamycin antibiotics. This study showed that constitutive and inducible resistance was found higher amongst MRSA isolates as compared to MSSA (Table 5). The ICR or $\mathrm{D}$ test positive $S$. aureus was shown in (Fig. 6).

\section{Grading of MRSA}

28(71.8\%) MRSA isolates were multi-resistant oxacillin resistant Staphylococcus aureus (MORSA) i.e. MDR strains exhibiting resistant to $\geq 3$ non- $\beta$-lactam antibiotics, and 11(28.2\%) MRSA isolates were nonmulti-resistant oxacillin resistant Staphylococcus aureus (NORSA) that were resistant to $\leq 2$ non $\beta$-lactam antibiotics. Association of multi-resistant observed with methicillin resistance is significant with $(p=0.03)$ at $95 \% \mathrm{Cl}$ (Table 6).

\section{Discussion}

S. aureus infection brought more health burden settings in low and middle-income countries. MRSA is emerging as a serious threat to public health worldwide. MRSA has significantly increased morbidity and mortality rate of patients along with the increased length of hospital stay. $S$. aureus infections are the significant clinical problem in medical practice as the organism showed resistance to the commonly used first-line drugs. The prevalence of MRSA, however, varies markedly country wise. In this study, the occurrence of $S$. aureus was studied among pediatric patients visiting IFCH from various samples. All the clinically $S$. aureus detected samples may serve as a reservoir of MRSA which may transmit the infection in a community. Thus, there is a chance of a rapid increase in the development of CAMRSA infection. Though infected persons with $S$. aureus are direct vital source whereas, they are also reported to be isolated from fomites [21]. Fomites can be considered as the indirect transmission route for $S$. aureus as well as other infectious diseases in hospital [22]. So, environmental sources and fomites cannot be ignored.

In the present study, 52(17.3\%) were found $S$. aureus isolates out of 300 culture positive samples. The result has quite resembled with the study carried out by Mukhiya et al (2012) with growth positivity of 
$17.4 \%$ [23]. Here the result also shows children are more prone to get $S$. aureus associated infection. It might be due to high chances of contact with an infectious agent or their low immunity. This finding shows that $S$. aureus is a major cause of pyogenic infections. The SST was the most common site for the infection because this bacterium normally inhabits in a skin. Pandey et al. (2012) reportedthe higher isolation of $S$. aureus from pus and wound $(78.37 \%)$ in comparison to blood $(17.11 \%)$ and urine $(4.50 \%)$ out of $111 \mathrm{~S}$. aureus consisting clinical samples [24]. The study also shows a parallel result, where $34(65.4 \%)$ S. aureus isolates were found in SST sample which is more than the samples obtained from RTIs, blood, and UTIs sample.

In our study, when comparing the number and percentages of $S$. aureus in Inpatient Department (IPD) and Outpatient Department (OPD), the rate of $S$. aureus in the present study was higher in inpatients (55.7\%) than compared to outpatients (44.3\%). Our result is in accordance with Sanjana et al (2010) in Nepal [25]. She also reported the higher prevalence of $S$. aureus among inpatient setting accounting $86(62.3 \%)$ as compared to outpatients 52(37.7\%). Likewise, if we look the distribution of MRSA isolates in different types of patients we can found them inpatients $21(40.4 \%)$ out of total 52 patients and outpatients $18(30.6 \%)$ out of total 52 patients. These findings also indicate the higher prevalence of nosocomial infections. This higher occurrence of MRSA among inpatients could be due to various hospital associated risk factors such as prolonged hospital stay, antibiotic treatment, underlying immunecompromised condition, hospital environment, instrumentation, and use of other invasive devices which predispose patients to MRSA acquisition.

Over the course of time, $S$. aureus has developed resistant to different antibiotics, which are used conventionally. All the MRSA isolates were resistant to more antibiotics than MSSA isolates. A significant difference $(p=0.866)$ was observed in the case of cefoxitin, gentamicin, amikacin, co-trimoxazole, and erythromycin. However, the difference observed in the case of tetracycline and ciprofloxacin was statistically insignificance ( $p>0.05)$. Significant MDR cases of MRSA were $28(71.8 \%)$ also found. It also showed high resistance to co-trimoxazole and erythromycin that accounts 56.4 and $53.9 \%$ respectively. These two antibiotics are usually used at random to cure a generalized and pyogenic infection. On the other hand, only $15.4 \%$ of MRSA isolates shown ICR. The increasing rate of Staphylococcal infections among the patients and the changing patterns of antimicrobial resistance has led to a renewed interest in the use of clindamycin therapy in treating such infections [26]. The overall findings suggest the alarming situation of antimicrobial resistance accompanied by $S$. aureus. The development of antibiotic resistance properties in the microbes are troublesome and has been described as a serious public health concern particularly in developing countries [27].

The study showed the frequency of MRSA to be 39(75\%). In another study carried out in Kathmandu valley by Shrestha et al (2009a and 2009b) reported $44.9 \%$ of MRSA from nosocomial S. aureus [28, 29]. Sanjana et al (2010) also reported 348(39.6\%) MRSA isolates at CMS-teaching Hospital [30]. Meanwhile, Rijal et al (2008) in Pokhara Valley reported 75.5\% MRSA isolate and similar report (69.1\%) MRSA isolate was found by Tiwari et al (2009) in western parts of Nepal [31, 32]. Which the authors have attributed to the indiscriminate use of antibiotics and its accessibility. 
Prompt detection of pathogens is necessary for detection and identification of the MRSA may control the severe epidemic. Currently, various molecular techniques are used but they are not economical and are unfeasible at small scale [33,34]. Availability of new drugs and inadequate establishment for the MRSA treatment brings the use of antibiotics combinations. Sometimes new treatments and therapy are not available against MRSA even in developed countries. For MDR S. aureus strains, a combination of different antibiotics seems the only option. Tetracycline/Sulbactam/Cefoperazone combination has shown better results than the old combination of Ampicillin/Sulbactam/Cefoperazone antibiotic [22]. Which is also, supported by similar MIC results of other group's data too [35]. Some variations occur may be due to differences in infection control measures, antibiotics prophylaxis, empirical therapy, treatments used in each ward and not less important, the clonal and often epidemic nature of these microorganisms.

The high rate of MRSA isolation and resistance to penicillin, ampicillin, cloxacillin, ciprofloxacin, gentamicin, erythromycin, clindamycin, co-trimoxazole, chloramphenicol etc. shows these antibacterial agents would be unreliable. Vancomycin seems to be the only antimicrobial agent which showed $100 \%$ sensitivity against $S$. aureus in the study. Hence, vancomycin may be used as the drug of choice for treating MDR-MRSA infections. However, frequent monitoring of vancomycin sensitivity and routine testing should be carried out. Use of vancomycin should be limited to preserve its value. It should be administered only in those cases where there are clearly needed. Although, all isolates were found sensitive to vancomycin, the screening test, and MIC determinations are recommended for early detection of impending resistance and monitoring the response to therapy. The regular surveillance of nosocomial infections including monitoring antibiogram of MRSA and MSSA and formulation of definite antibiotic policy may be helpful in reducing the incidence of MRSA infection. The study is an opening to facilitate epidemiological studies.

However, apart from the combination of the antibiotics for the treatment of MRSA, new treatment options of using other antibiotics are slowly emerging. Nevertheless, it has its own drawback like drug supply, and its cost as well as resistance itself. Therefore, it is not successfully being enforced in practice. Even though the use of new antibiotics offers the different antibiotic resistance mechanisms to the organisms and new resistance properties will emerge latterly. Thus the use of affordable antibiotics in suitable combinations is only promising alternatives for developing nations. The authors recommended detecting novel resistance mechanisms such as mecC or uncommon phenotypes such as borderline-resistant oxacillin resistant.

\section{Conclusions}

The occurrence of MRSA was found to be $(n=39,75.0 \%)$ out of total of 52 S. aureus isolates. The highest number of MRSA isolates was found in the toddler age group of the male patient than the female patient. Methicillin susceptibility of $S$. aureus was found higher in inpatient than outpatient. Moreover, most of the MRSA isolates were obtained from SSTIs than other samples. MRSA found to be less resistant to gentamicin, cloxacillin, and clindamycin so these antimicrobial agents can be also used like mupirocin for the treatment of skin and soft tissue infections (SSTIs) and decolonization of MRSA carriers but the 
use such drugs should be limited. Alternatively, drugs like trimethoprim-sulfamethoxazole (TMP-SMX), teicoplanin, linezolid, quinupristin/dalfopristin, rifamycin, vancomycin etc. or with suitable combination drugs are prescribed for MRSA treatment.

Both cefoxitin disc diffusion and D-test are the easy, reliable and cost-effective method for detection of methicillin and inducible $\mathrm{MLS}_{\mathrm{B}}\left(\mathrm{iMLS}_{\mathrm{B}}\right.$ ) resistance respectively. Cefoxitin test and ICR (D-test) should be made compulsory in all $S$. aureus isolates in addition to the routine susceptibility test method. It is because the routine susceptibility test cannot detect MRSA unless cefoxitin disc is used. Likewise, inducible $\mathrm{MLS}_{\mathrm{B}}$ resistance is not detected unless erythromycin and clindamycin antibiotics are placed 15$26 \mathrm{~mm}$ apart. Although they are time-consuming and labor-intensive methods. The sensitivity and specificity of these methods are also low and less reliable than compare to other detection methods like minimum inhibitory concentration [36] determination, molecular techniques etc. Though $\mathrm{iMLS}_{\mathrm{B}}$ resistance is not associated with MRSA and widespread use of $M L S_{B}$ has led to increasing resistance to $M L S_{B}$ antibiotics, they are still used to treat infectionsassociatedwith $S$. aureus. It is also because of safety and easy availability of these drugs compare to newer classes antibiotics.

The study shows the MRSA occurrence is prevalent in pediatric patients. This corroborates the findings of previous researchers referred to the text. Nevertheless, the total number of $S$. aureus isolates and 6 months of study duration are very less, which might be a possible source of errors to draw definite conclusions. Newer classes of drugs are found effective than $\beta$-lactam drugs to treat $S$. aureus infection. Moreover, MRSA infection is still one of the most life-threatening infections in hospitals. Therefore, regular surveillance of MRSA shall be carried out in all hospital settings. In addition, restriction on the indiscriminate use of such drugs may be an effective strategy to control the drug resistance. We should discourage empirical therapy practices and consider microbiological test reports. This might be an effective strategy to tackle AMR problem and improve the infection treatment plan.

\section{Limitations}

The study was limited by molecular test for genotyping or SCCmec typing and similarly, Minimum Inhibitory Concentration (MIC) test for MRSA confirmatory was not done due to lack of resources and time-bound factors. Neither risk factor analysis of MRSA carrier nor longitudinal data of MRSA trend was

generated due to the limitation of current study settings and expertise in our network. Moreover, the study was conducted to a single hospital, so the findings do not represent a broad population and non-patients.

\section{Abbreviations}

AST: Antibiotic susceptibility test; ATCC: American Type Culture Collection; BA: Blood Agar; CLSI: Clinical Laboratory Standard Institute; IFCH: International Friendship Children Hospital; MDR: Multiple Drug Resistance; $\mathrm{MLS}_{\mathrm{B}}$ : Macrolide Lincosamide Streptogramin-B; BHI: Brain Heart Infusion broth; MSA: Mannitol Salt Agar; SSTIs: Skin and soft-tissue infections; Toxic Shock Syndrome (TSS); ZOI: Zone of Inhibition, ICR: Inducible Clindamycin Resistant (ICR) test or D-zone test; AMR: Antimicrobial Resistance 


\section{Declarations}

\section{Ethics approval and consent to participate}

At International Friendship Children Hospital (IFCH), all MRSA strains are routinely collected in the microbiology laboratory and stored at $-70^{\circ} \mathrm{C}$. The current study thus is descriptive of a bacterial collection of those isolates. No patient-related data were collected. Ethical approval was therefore not required. The study was a laboratory-based basic science study. Informed written consent was not applicable since this study was not directly involved with patients.

\section{Consent for publication}

Not applicable

\section{Availability of data and materials}

All data generated and analyzed during this study are deposited in the public data repository. Figshare [https://doi.org/10.6084/m9.figshare.8203877].

\section{Competing interests}

The authors declare that they have no competing interests.

\section{Funding}

This study conducted without specific grant from any funding agency in the public, commercial or nonprofit sector.

\section{Authors' contributions}

R.R.G. conceived the study, reviewed the literature, performed the experiments, compiled, submitted and communicated the article. P.M. provided technical consultation on planning experiments, drafting of the manuscript and also helped to article formatting, data acquisition, analysis, interpretation, and quality assurance. G.GC. was involved in the conception and design of the study, supervised the study, revision 
of the article and helped to write a final version of the manuscript. All authors made substantial contributions to editing and writing the manuscript. All authors read and approved the final manuscript.

\section{Author details}

1. Raja Ram Gurung: Tri-Chandra Multiple Campus, Ghantaghar, Kathmandu Nepal and Biotechnology Society of Nepal (BSN), Ranidevimarg, Kathmandu Nepal

2. Ganga GC: Asst. Professor, Department of Microbiology, Tri-Chandra Multiple Campus, Ghantaghar, Kathmandu Nepal

3. Prashanna Maharjan: Amrit Science Campus, Thamel, Kathmandu, Nepal and Biotechnology Society of Nepal (BSN), Ranidevimarg, Kathmandu Nepal

\section{Acknowledgements}

The authors would like to express sincere thanks to all the personnel of Microbiology Laboratory especially Dr. Shovana Thapa (Pathologist) and Mr. Amar Twayana (Laboratory In-charge) at International Children Friendship Hospital (IFCH), Maharajgung to conduct this study and Department of Microbiology at TC. Additionally, we would like to thank Roshan Lal Shrestha, PhD from NIH, USA for his valuable advice and suggestions in proofreading of the manuscript.

\section{References}

1. Friendship B, Weese S: Methicillin resistant Staphylococcus aureus (MRSA). Advances in Pork Production 2009, 20:173-180.

2. Kluytman J, Belkum AV, Verbrugh H: Nasal carriage of Staphylococcus aureus: epidemiology, underlying mechanisms, and associated risks. Clin Microbiol rev 1997, 10(3):505-520.

3. Tortora GJ, Funke BR, case CL: Microbiology- An Introduction, 10 edn. San Francisco: Pearson Education Inc.; 2010.

4. Presscott L, Harley J, lein D: Textbook of Microbiology, 5th ed edn. USA: Brown Publishers; 2002.

5. Tille PM: Catalase-positive, gram-positive cocci., 13th edn. St. Louis, USA: Mosby Elsevier; 2014.

6. Grundmann H, Aires-de-Sousa M, Boyce J, Tiemersma E: Emergence and resurgence of meticillinresistant Staphylococcus aureus as a public-health threat. Lancet 2006, 368(9538):874-885.

7. Krishnamurthy V, Saha A, Renushri BV, Nagaraj ER: Methicillin resistant Staphylococcus aureus carriage, antibiotic resistance and molecular pathogenicity among healthy individuals exposed and not exposed to hospital environment. J Clin Diagn Res 2014, 8(7).

8. Fomda BA, Thokar MA, Khan A, Bhat JA, Zahoor D, Bashir G, Majid A, Ray P: Nasal carriage of Methicillin-resistant Staphylococcus aureus among healthy population of Kashmir, India. Indian J 
Med Microbiol 2014, 32(1):39-43.

9. Cosgrove SE, Qi Y, Kaye KS, Harbarth S, Karchmer AW, Carmeli Y: The impact of Methicillin resistance in Staphylococcus aureus bacteremia on patient outcomes: mortality, length of stay, and hospital charges. Infect Control Hosp Epidemiol 2005, 26(2):166-174.

10. Engemann JJ, Carmeli Y, Cosgrove SE, Fowle VG, Bronstein MZ, Trivette SL, Briggs JP, Sexton DJ, Kaye KS: Adverse clinical and economic outcomes attributable to Methicillin resistance among patients with Staphylococcus aureus surgical site infection. Clin Infect Dis 2003, 1:592-598.

11. Antimicrobial resistance [https://www.who.int/news-room/fact-sheets/detail/antimicrobialresistance]

12. Burian M, Wolz C, Goerke C: Regulatory adaptation of Staphylococcus aureus during nasal colonization of humans. PLOS ONE 2010, 5(4):e10040.

13. Lowy FD: Staphylococcus aureus infections. N Engl J Med 1998, 339(8):520-532.

14. Chambers HF: The changing epidemiology of Staphylococcus aureus? Emerg Infect Dis 2001, 7(2):178-182.

15. Lowy FD: Antimicrobial resistance: the example of Staphylococcus aureus. J Clin Invest 2003, 111(9):1265-1273.

16. Chaturvedi P, Singh AK, Shukla S, Agarwal L: Prevalence of Mupirocin Resistant Staphylococcus aureus Isolates among patients admitted to a Tertiary care hospital. N Am J Med Sci 2014, 6(8):403407.

17. Lu PL, Chin LC, Peng CF, Chiang YH, Chen TP, Ma L, Siu LK: Risk factors and molecular analysis of community methicillin-resistant Staphylococcus aureus carriage. J Clin Microbiol 2005, 43(1):132139.

18. Hussain FM, Boyle-Vavra S, Daum RS: Community-acquired methicillin-resistant Staphylococcus aureus colonization in healthy children attending an outpatient pediatric clinic. Pediatr Infect Dis J 2001, 20(8):763-767.

19. CLSI: Performance standards for Antimicrobial Susceptibility Testing: Twenty-Fifth Informational Supplement. In. Wayne, USA: Clinical and Laboratory Standards Institute; 2016.

20. Natrajsetty HS, Vijayalakshmi IB, Narasimhan C, Manjunath CN: Purulent pericarditis with quadruple valve endocarditis. The American journal of case reports 2015, 16:236-239.

21. Neely AN, Maley MP: Survival of Enterococci and Staphylococci on hospital fabrics and plastic. $J$ Clin Microbiol 2000, 38(2):724-726.

22. Dudhagara PR, Ghelani AD, Patel RK: Phenotypic characterization and Antibiotics combination approach to control the Methicillin-resistant Staphylococcus aureus (MRSA) strains isolated from the hospital derived Fomites. AJMS 2011, 2:72-78.

23. Mukhiya RK, Shrestha A, Rai SK, Panta K, Singh RN, Rai G, Prajapati A: Prevalence of Methicillinresistant Staphylococcus aureus in hospitals of Kathmandu valley. NJST 2012, 13(2):185-190. 
24. Pandey S, Raza MS, Bhatta CP: Prevalence and antibiotic sensitivity pattern of methicillin- resistantStaphylococcus aureus in Kathmandu medical college -teaching hospital. JIOM 2012, 34(11):13-17.

25. Sanjana RK, Shah R, Chaudhary N, Singh YI: Prevalence and antimicrobial susceptibility pattern of methicillin-resistant Staphylococcus aureus (MRSA) in CMS-teaching hospital: a preliminary report. JCMS-Nepal 2010, 6(1):1-6.

26. Aleksandra AD, Misic MS, Mira ZV, Violeta NM, Dragana IT, Zoran BM, Dejan VS, Milanko SD, Dejan BD: Prevalence of inducible clindamycin resistance among community-associated staphylococcal isolates in central Serbia. Indian J Med Microbio/ 2014, 32(1):49-52.

27. Vandepitte J, Verhaegen J, Engbaek K, Rohner P, Piot P, Heuck CC: Basic laboratory procedure in clinical bacteriology. In., 2 edn. Geneva: World Heath Organisation; 2003.

28. Shrestha B, Pokhrel B, Mohapatra T: Antibiotic susceptibility pattern of nosocomial isolates of Staphylococcus aureus in a tertiary care hospital, Nepal. J Nepal Med Assoc 2009 48(175):234-238.

29. Shrestha B, Pokhrel BM, Mohapatra TM: Phenotypic characterization of nosocomial isolates of Staphylococcus aureus with reference to MRSA. J Infect Dev Ctries 2009, 3(7):554-560.

30. Rajbhandari R, Manandhar S, Shrestha J: Comparative study of MRSA and its antibiotic susceptibility pattern in indoor and outdoor patients in Bir Hospital, Nepal. Nepalese J Microbiol $2003,1: 62-65$.

31. Rijal KR, Shrestha N, Pahari N, Shrestha B, Paudel B, Nepal A, Ghimire P, Rijal B: Methicillin Resistant Staphylococcus aureus in patients visiting western regional hospital, Pokhara. JIOM 2008, 30(1):2125.

32. Tiwari HK, Das AK, Sapkota D, Sivarajan K, Pahwa VK: Methicillin resistant Staphylococcus aureus: prevalence and antibiogram in a tertiary care hospital in western Nepal,. J Infect Dev Ctries 2009, 3(9):681-684.

33. Fang G, Keys TF, Gentry LO, Harris AA, Rivera N, Getz K, Fuchs PC, Gustafson M, Wong ES, Goetz A et al: Prosthetic valve endocarditis resulting from Nosocomial Bacteremia. Ann Intern Med 1993, 119(7):560-567.

34. Tan TY, Corden S, Barnes R, Cookson B: Rapid identification of methicillin-resistant Staphylococcus aureus from positive blood cultures by real-time fluorescence PCR. J Clin Microbio/ 2001, 39(12):4529-4531.

35. Gad GFM, El-Ghafar AE-GFA, El-Domany RAA, Hashem ZS: Epidemiology and antimicrobial resistance of Staphylococcal isolated from different infectious diseases. Braz J Microbiol 2009, 41:333-344.

36. Brock TD, Madigan MT, Martinko JM: Brock Biology of microorganisms, 10 edn: Pearson Prentice Hall; 2005.

\section{Tables}

Table 1 Distribution of $S$. aureus in different age groups and types of patients 


\begin{tabular}{|c|c|c|c|c|c|c|c|}
\hline \multirow[t]{3}{*}{ Age group } & \multirow[t]{3}{*}{ Age ranges } & \multirow{2}{*}{\multicolumn{4}{|c|}{$\frac{\text { Types of patients }}{\text { Outpatients Inpatients }}$}} & \multirow{2}{*}{\multicolumn{2}{|c|}{ Total }} \\
\hline & & & & & & & \\
\hline & & No. & $\%$ & No. & $\%$ & No. & $\%$ \\
\hline Neonate & Newborn up to first 28 days & 53 & 5.8 & 4.0 & 7.7 & 7 & 13.5 \\
\hline Infant & 28 days - below 1 years & 4 & 7.7 & 5.0 & 9.6 & 9 & 17.3 \\
\hline Toddlers & 1 year - below 3 year & 7 & 13.5 & 10.0 & 19.2 & 17 & 32.7 \\
\hline \multicolumn{2}{|c|}{ Pre-School 3 years - below 5 years } & 6 & 11.5 & 4.0 & 7.7 & 10 & 19.2 \\
\hline School & 6 years- 10 years & 3 & 5.8 & 4.0 & 7.7 & 7 & 13.5 \\
\hline \multicolumn{2}{|c|}{ Adolescent11 years- below 15 years } & 0 & 0.0 & 2.0 & 3.8 & 2 & 3.8 \\
\hline \multicolumn{2}{|l|}{ Total } & 23 & 44.3 & 29 & 55.7 & 52 & 100.0 \\
\hline
\end{tabular}

Table 2 Distribution of MRSA in outpatients and inpatients

\begin{tabular}{ccccc}
\hline Methicillin & In-patients & Out-patients & Total No. (\%) & p-value \\
susceptibility & No. (\%) & No. (\%) & & \\
\hline MRSA & $21(72.4)$ & $18(78.3)$ & $39(75.0)$ & p>0.05 \\
MISA & $5(17.2)$ & $2(8.7)$ & $7(13.5)$ & \\
MSSA & $3(10.3)$ & $3(13.0)$ & $6(11.5)$ & \\
Total No. (\%) & $29(100.0)$ & $23(100.0)$ & $52(100.0)$ & \\
\hline
\end{tabular}

Table 3: Gender wise distribution of $S$. aureus in various infection groups

\begin{tabular}{ccccccc}
\hline $\begin{array}{c}\text { Infection } \\
\text { group }\end{array}$ & $\begin{array}{c}\text { Male } \\
\text { No. }\end{array}$ & $\begin{array}{c}\text { Male } \\
(\%)\end{array}$ & $\begin{array}{c}\text { Female } \\
\text { No. }\end{array}$ & $\begin{array}{c}\text { Female } \\
(\%)\end{array}$ & $\begin{array}{c}\text { Total No. of } S . \\
\text { aureus }\end{array}$ & $\begin{array}{c}\text { Total \% of } S . \\
\text { aureus }\end{array}$ \\
\hline SSTIs & 23 & 44.2 & 11 & 21.2 & 34 & 65.4 \\
RTIs & 7 & 13.5 & 3 & 5.8 & 10 & 19.2 \\
Blood & 5 & 9.6 & 2 & 3.9 & 7 & 13.5 \\
UTIs & 0 & 0 & 1 & 1.9 & 1 & 1.9 \\
Total & 35 & 67.3 & 17 & 32.7 & 52 & 100.0 \\
\hline
\end{tabular}

Table 4: Antibiogram of MRSA and MSSA 


\begin{tabular}{|c|c|c|c|c|c|c|}
\hline \multirow[t]{2}{*}{ Antibiotics used $(\mu \mathrm{g})$} & \multicolumn{2}{|c|}{$\operatorname{MSSA}(n=13)$} & \multicolumn{2}{|c|}{$\operatorname{MRSA}(\mathrm{n}=39)$} & \multirow{2}{*}{$\begin{array}{c}\text { Total } \\
\text { Sensitive } \\
\mathrm{N}(\%)\end{array}$} & \multirow{2}{*}{$\begin{array}{c}\text { Total Resistant } \\
\mathrm{N}(\%)\end{array}$} \\
\hline & $\mathrm{S}[\mathrm{n}(\%)]$ & $\mathrm{R}[\mathrm{n}(\%)]$ & $\mathrm{S}[\mathrm{n}(\%)]$ & $\mathrm{R}[\mathrm{n}(\%)]$ & & \\
\hline Cefoxitin (CX 30) & $0(0.0)$ & $13(100.0)$ & $0(0.0)$ & $39(100.0)$ & $13(25.0)$ & $39(75.0)$ \\
\hline Penicillin (P 10) & $0(0.0)$ & $13(100.0)$ & $0(0.0)$ & $39(100.0)$ & $13(25.0)$ & $39(75.0)$ \\
\hline Vancomycin (VA 5) & $13(100.0)$ & $0(0.0)$ & $39(100.0)$ & $0(0.0)$ & $52(100.0)$ & $0(0.0)$ \\
\hline Cloxacillin (COX 5) & $10(76.9)$ & $3(23.1)$ & $32(82.1)$ & $7(17.9)$ & $42(80.8)$ & $9(19.2)$ \\
\hline Ampicillin (AMP 10) & $4(30.8)$ & $9(69.2)$ & $14(35.9)$ & $25(64.1)$ & $18(34.6)$ & $34(65.4)$ \\
\hline Ciprofloxacin (CIP 5) & $2(15.4)$ & $11(84.6)$ & $18(46.2)$ & 21(53.9) & $20(38.5)$ & $32(61.5)$ \\
\hline $\begin{array}{l}\text { Co-trimoxazole } \\
\text { (COT } 1.25 / 23.75)\end{array}$ & $9(69.2)$ & $4(30.8)$ & $22(56.4)$ & $17(43.6)$ & $31(59.6)$ & $21(40.04)$ \\
\hline Erythromycin (E 15) & $7(53.9)$ & $6(46.2)$ & 21(53.9) & $18(46.2)$ & 28(53.8) & $24(46.2)$ \\
\hline Clindamycin (CD 2) & $10(76.9)$ & $3(23.1)$ & $29(74.1)$ & $10(25.6)$ & $39((75.0)$ & $13(25.0)$ \\
\hline Gentamycin (GEN 10) & 13(100.0) & $0(0.0)$ & $35(89.9)$ & $4(10.2)$ & $48(92.3)$ & $4(7.7)$ \\
\hline $\begin{array}{l}\text { Chloramphenicol (C } \\
\text { 30) }\end{array}$ & 11(84.6) & $3(23.1)$ & $30(76.9)$ & $9(23.1)$ & $41(78.8)$ & $12(21.2)$ \\
\hline Azithromycin (AZM 15) & $8(61.5)$ & $5(38.5)$ & $10(25.6)$ & $29(74.4)$ & $18(34.6)$ & $34(65.4)$ \\
\hline Tetracycline (TE 30) & $12(92.3)$ & $0(0.0)$ & $19(48.7)$ & $20(51.3)$ & $31(59.6)$ & $20(40.4)$ \\
\hline
\end{tabular}

Table 5: Susceptibility pattern towards erythromycin and clindamycin

\begin{tabular}{|c|c|c|c|c|c|c|}
\hline $\begin{array}{l}\text { Resistant and } \\
\text { susceptible } \\
\text { phenotype }\end{array}$ & Erythromycin & Clindamycin & D- test & $\begin{array}{l}\text { S. aureus } \\
\text { No. (\%) }\end{array}$ & $\begin{array}{c}\text { MRSA } \\
\text { No. (\%) }\end{array}$ & $\begin{array}{c}\text { MSSA } \\
\text { No. (\%) }\end{array}$ \\
\hline Inducible $\mathrm{MLS}_{\mathrm{B}}$ & $\mathrm{R}$ & $S$ & $\mathrm{D}+$ & $8(15.4)$ & $6(15.4)$ & $2(15.4)$ \\
\hline Constitutive $\mathrm{MLS}_{\mathrm{B}}$ & $\mathrm{R}$ & $\mathrm{R}$ & - & $7(13.5)$ & $5(12.8)$ & $2(15.4)$ \\
\hline $\mathrm{MS}_{\mathrm{B}}$ & $\mathrm{R}$ & S & D- & $17(32.7)$ & $15(38.5)$ & $4(30.8)$ \\
\hline Susceptible & S & S & - & $20(38.5)$ & $13(33.3)$ & $5(38.5)$ \\
\hline Total no. (\%) & NA & NA & NA & $52(100)$ & 39 (100) & $13(100)$ \\
\hline
\end{tabular}

$\mathrm{iMLS}_{\mathrm{B}}=$ inducible $\mathrm{iMLS}_{\mathrm{B}}$ phenotype or ICR phenotypes, $\mathrm{CMLS}_{\mathrm{B}}=$ Constitutive $\mathrm{MLS}_{\mathrm{B}}$ phenotype, $\mathrm{MS}_{\mathrm{B}}=\mathrm{MS}_{\mathrm{B}}$ phenotype

Table 6: Multi drug Resistance pattern of MRSA

\begin{tabular}{ccccc}
\hline \multicolumn{3}{c}{ Multi-drug resistant isolates } & Total & p-value \\
MRSA & MORSA & NORSA & & \\
\hline Count & 28 & 11 & 39 & $\mathrm{p}<0.05$ \\
$\%$ & $71.8 \%$ & $28.2 \%$ & $100.0 \%$ & \\
\hline
\end{tabular}




\section{Figures}

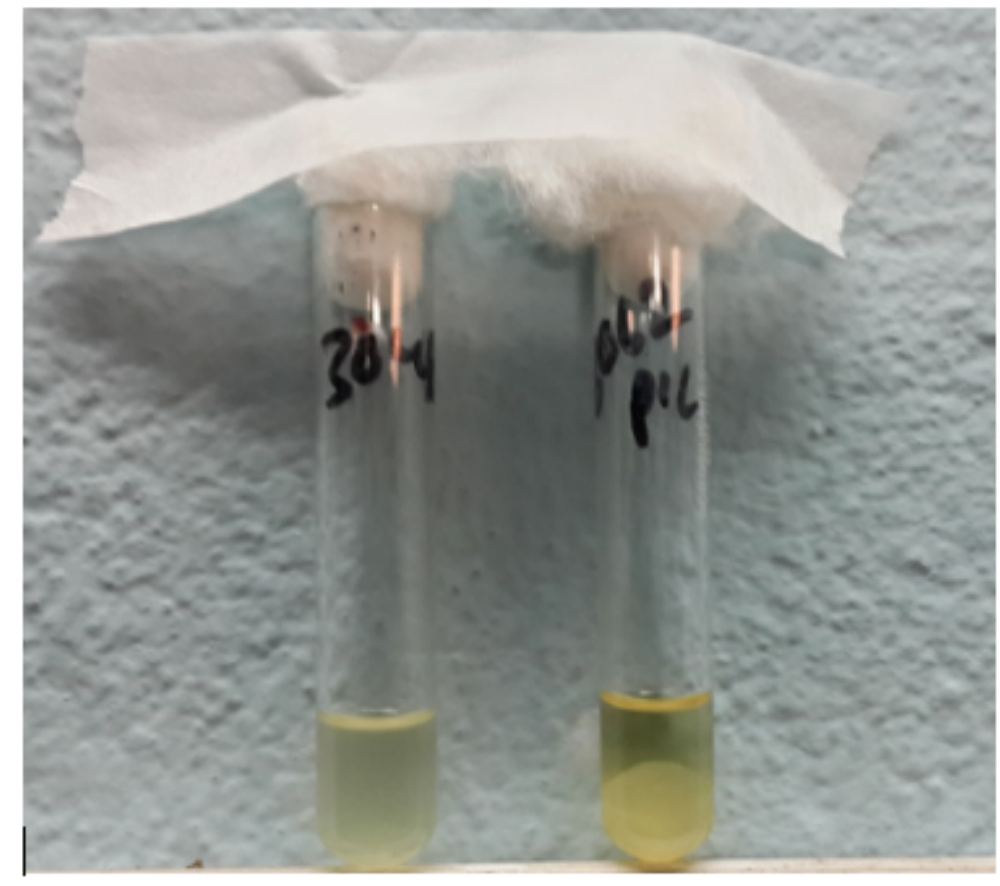

Figure 1

Photograph of Tube coagulase test results (a) - ve and (b) + ve

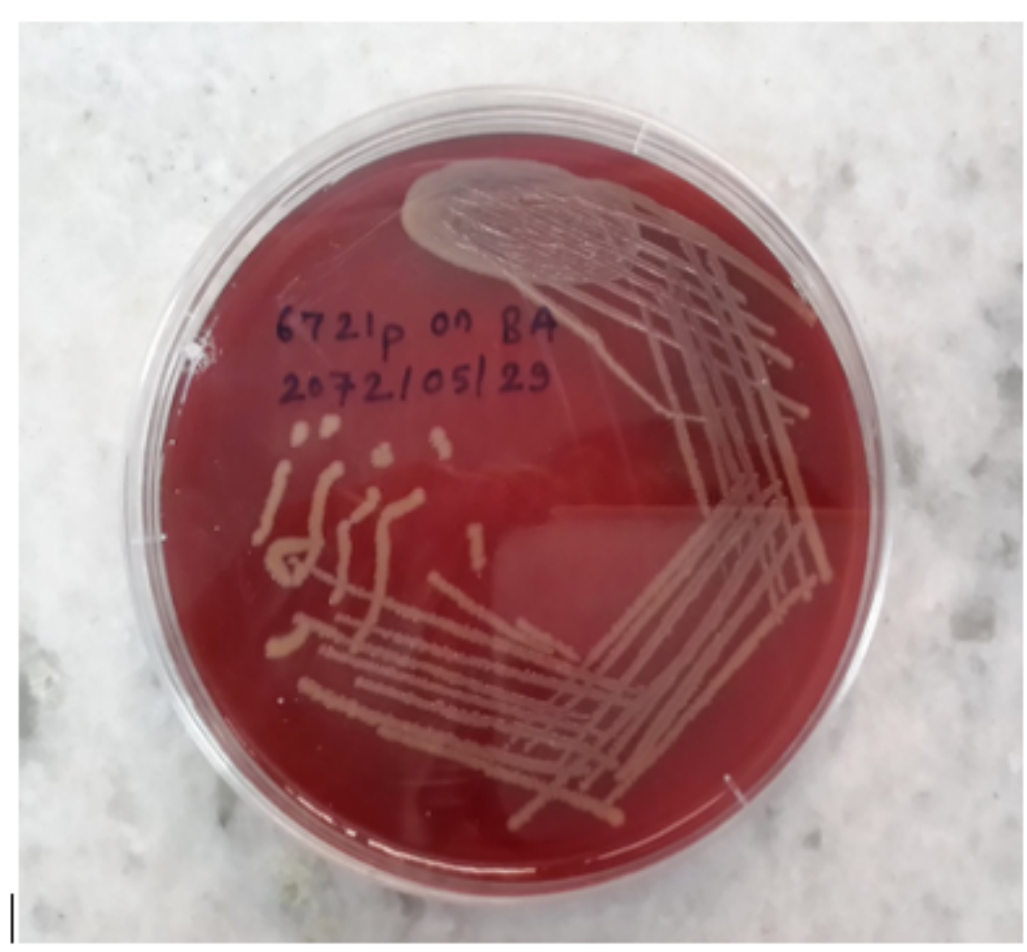

Figure 2 
Photograph of S. aureus in BA media

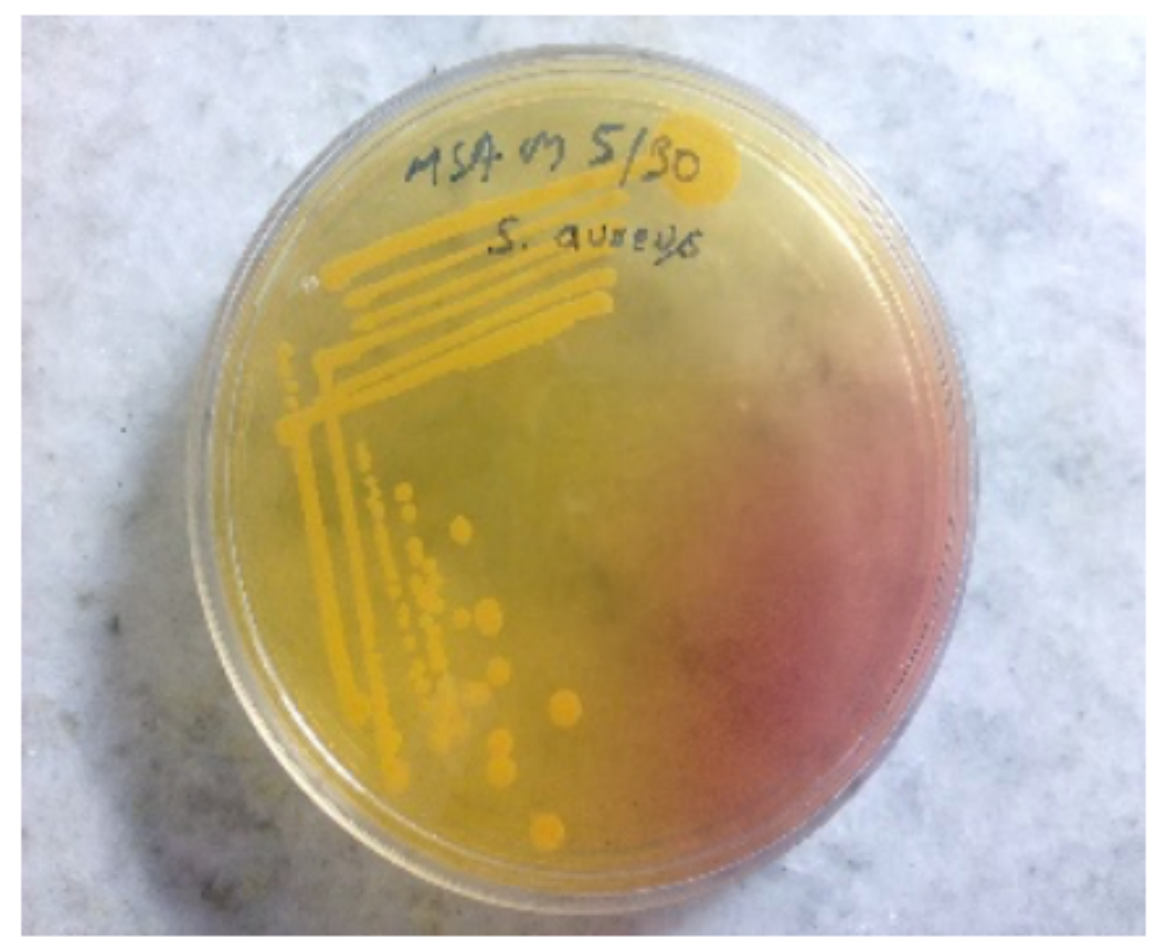

Figure 3

Photograph of S. aureus in MSA media

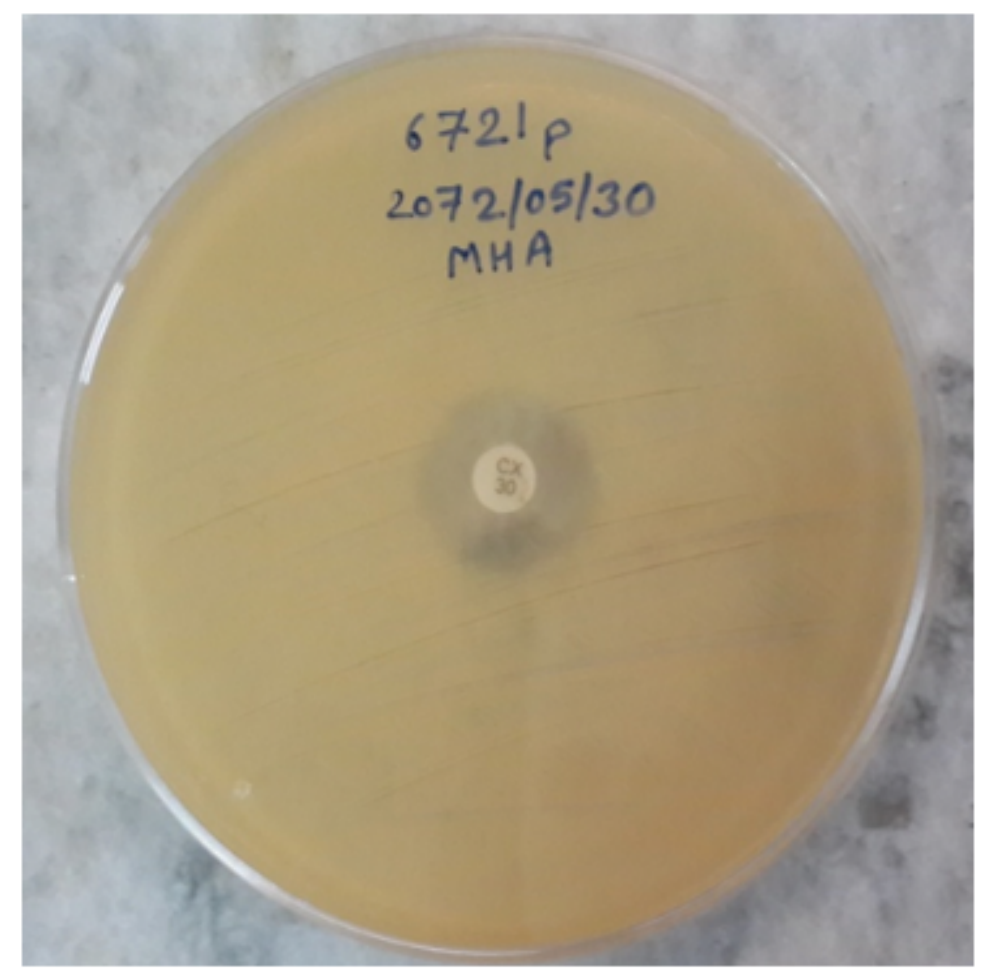

Figure 4 
Photograph of S. aureus in MHA media showing methicillin resistance character against cefoxitin disc

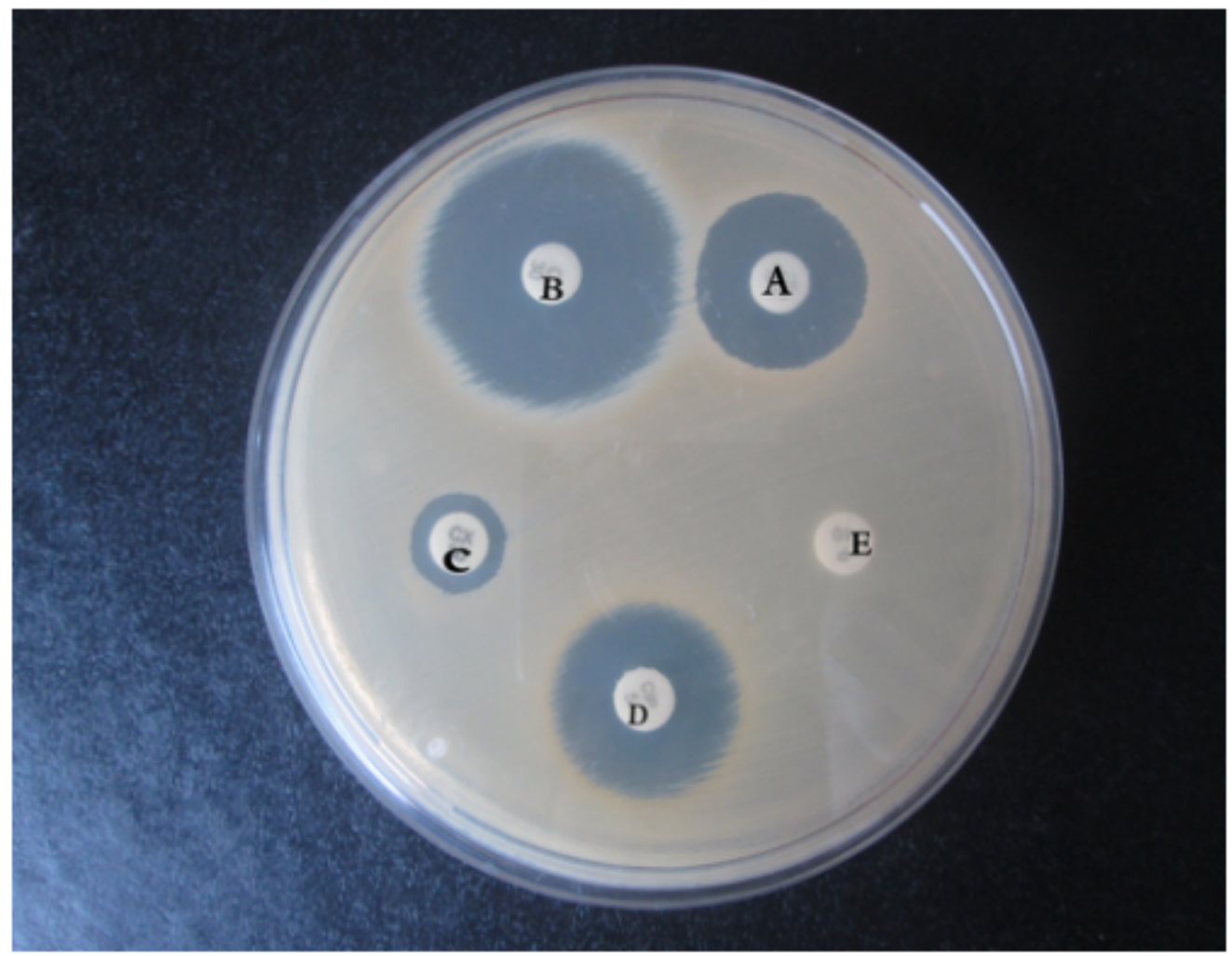

Figure 5

Photograph of AST of MRSA in MHA medium against 1st line drugs 


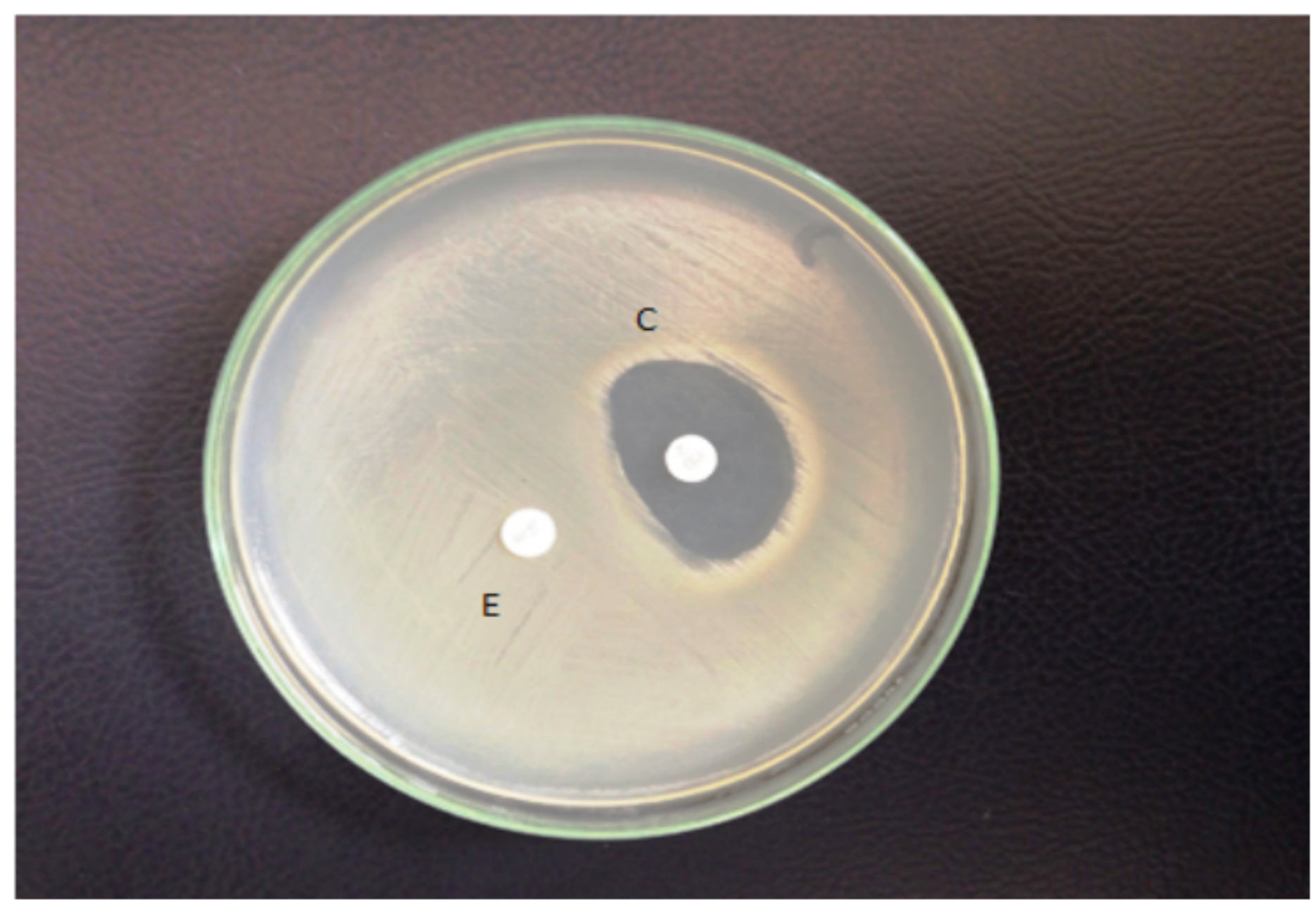

Figure 6

Photograph of ICR (D-test) positive showing MRSA in MHA media

\section{Supplementary Files}

This is a list of supplementary files associated with this preprint. Click to download.

- AdditionalFile8SupportingdatainExcelform.xlsx 